\title{
Vertebrobasilar vasospasm after aneurysmal subarachnoid hemorrhage: review
}

\begin{abstract}
In the present review we outline the clinical data, diagnosis and prognosis of vertebrobasilar (VB) vasospasm (VS) after subarachnoid hemorrhage (SAH). Aneurismal SAH is associated with high rates of morbidity and mortality. Although a major advancement has been made in recent years in diagnosis and treatment of aneurysmal SAH, $30 \%$ of the patients who survived the initial bleeding show further deterioration as a results of delayed cerebral ischemia (DCI) that traditionally was associated with large arteries lasting vasospasm (VS). The diagnosis of cerebral VS is based on clinical presentation, transcranial Doppler (TCD) evaluation and perfusion imagines as significant arterial narrowing is associated with reduced cerebral perfusion in the affected territories, DCI and cerebral infarcts. Although cerebral VS in the anterior circulation was intensely studied, little is known about VS in the posterior cerebral circulation.

The incidence of posterior circulation VS and basilar artery (BA) VS is lower than the incidence reported for the anterior circulation, however it is associated with worst outcome. The diagnosis of posterior circulation and BA-VS is mainly based on measurement of TCD intracranial/extracranial (IC/EC) flow velocities FVs ratio that have been correlated with arterial narrowing on computerized tomography (CT) angiography, cerebellar hypoperfusion and outcome. Within the past decade, there has been very little scientific activity to follow up on the research outlined above although TCD grading criteria for BAVS are in widely used on daily clinical practice for the diagnosis of posterior circulation VS. The purpose of the present review is to increase clinician awareness and knowledge of posterior circulation VS after aneurysmal SAH and other intracranial pathology as for it major impact on outcome of patients with cerebral VS.
\end{abstract}

Keywords: Vasospasm, Subarachnoid hemorrhage, Transcranial Doppler, Basilar artery, Cerebral Blood flow, Outcome
Volume 8 Issue I - 2018

\section{Gavin W Britz,' Gill E Sviri²}

'Department of Neurosurgery, Houston Methodist Hospital, USA

2Department of Neurosurgery, Rambam (Maimonides) Health Care Campus, The Technion, Israel Institute of Technology, Israel

Correspondence: Gill E Sviri, Department of Neurosurgery, Rambam (Maimonides) Health Care Campus, The Technion, Israel Institute of Technology, Haifa, Israel,

Email g_sviri@rambam.health.gov.il

Received: December 25, 2017 | Published: February 05, 2018
Abbreviations: VB, Vertebrobasilar; VS, Vasospasm; SAH, Subarachnoid Hemorrhage; DCI, Delayed Cerebral Ischemia; TCD, Transcranial Doppler; BA, Basilar Artery; CT, Computerized Tomography

\section{Introduction}

Aneurysmal subarachnoid hemorrhage (SAH) affects 5-10 per 100,000 individuals per year1 and is associated with high rates of morbidity and mortality. ${ }^{1-4}$ Main contributor to poor outcomes after SAH are the early cerebral injury (ECI) caused by the immediate increase in intracranial pressure, decreased cerebral perfusion pressure and global ischemia ${ }^{1,5-9}$ and delayed cerebral ischemia (DCI) which affects $30 \%$ of the SAH survivors leading to neurological deficit, cognitive decline, ${ }^{4,69-13}$ and death. ${ }^{14}$ This ischemia is historically though to results from a long-lasting narrowing of the large-capacity cerebral arteries. Kassell et al ${ }^{15}$ published statistics that roughly hold to this day, whereby $40-70 \%$ of aSAH patients having survived the acute phase demonstrated angiographic VS, 20-30\% manifested delayed neurologic deficits (DID), and 7\% having died as a consequence there of. Another seminal study by Broderick et al. ${ }^{16}$ have discerned a mortality rate of $45 \%$, attributed 2 of $36(6 \%)$ total deaths to VS, even though $44 \%$ of all patients manifested DCI. In 1994, Dorsch \& King ${ }^{17}$ published a review based on over 30,000 clinical cases that established an incidence of angiographic vasospasm at $43.3 \%$ overall and DID occurring at a rate of $32.5 \%$. Of those who experienced DID, $34 \%$ sustained permanent neurological deficits and 30\% died, such that VS was considered the cause of death in roughly $10 \%$ of aSAH patients.
Cerebral VS after aneurysmal SAH develops as soon as 3 to 4 days after the initial bleeding, with maximal narrowing occurring between days 5 and 14, gradually resolving by the third or fourth week. ${ }^{18-}$ 20 Symptomatic VS presents through focal neurological signs such as hemiparesis, aphasia, apraxia, neglect, or cranial nerve deficits; alternately it can manifest in a decreased level of consciousness, typically fluctuating and with gradual onset. ${ }^{13}$ Its clinical features are functionally determined by the involved ischemic territory. Unlike anterior circulation VS which have been studied and reported very intensively, vertebrobasilar (VB) VS has rarely been studied clinically. Therefore, many clinicians may not be well guided in the monitoring and treatment of VB-VS. The purpose of this review is to highlight the prevalence, diagnostic criteria and clinical importance of Basilar artery (BA) VS after aneurysmal SAH. In the present review we outline the clinical data, diagnosis and prognosis of vertebrobasilar vasospasm. The purpose of the present study is to increase clinician awareness and knowledge of posterior circulation VS after aneurysmal SAH and other intracranial pathology as for it major role in in outcome of patients with cerebral VS.

\section{Vertebrobasilar Vasospasm}

In contrast to the more robust scientific interest in and concentration on CVS of the anterior circulation, research on the diagnosis, clinical features and pathophysiological characteristics of posterior circulation VS was belated, largely owing to the unique methodologic difficulties and obscurities involved in the investigation thereof. In 1964 Crompton $^{21}$ reported bilateral VS in the posterior communicating arteries in one of his cases of basilar tip aneurysms. In 1968, Wilkins 
et al. ${ }^{22}$ had noted VS in the event of ruptured VB aneurysm, however the affected arteries were in the anterior circulation. In 1977, Saito et al. ${ }^{23}$ discovered VS of the VB system owing to aneurysmal SAH, albeit rare among its cohort Saitos group ${ }^{23}$ subsequently argued that VS of the VB system resulting from aneurysmal SAH presented with negligible neurological deficit relative to vasospasm of the anterior circulation. ${ }^{24}$ Yet in 1978, Marshall et al. ${ }^{25}$ reported on four patients who presented with significant head trauma and developed neurological deficits suggestive of primary brainstem or cerebellar dysfunction without an understanding of the underlying cause. The intracranial pressure had been normal in four of the patients and mildly elevated in two, indicating that the clinical presentation could not be explained on the basis of secondary compression of the brainstem and thus pointing towards an alternate etiology that was then clarified on angiography namely, VB-VS.

Research on the use of transcranial Doppler (TCD) for the diagnosis of VS took off in the 1980s. The landmark paper by Aaslid et al. ${ }^{26}$ in 1982, recorded normal flow velocities (FVs) by means of TCD, did note the detection of Doppler shift from the BA, yet a follow-up paper two years later on the sonographic diagnosis of VS neglected the VB system. ${ }^{27}$ However, as research on the use of TCD for assessment of intracranial arteries broke further ground, more attention was paid the posterior circulation. Lindegaard et al. ${ }^{28}$ in a study that illuminated the inverse relationship between residual lumen diameter and FVs, provided recordings of flow volumes in both angiographically normal as well as stenotic vessels including those of the BA. Moreover, featured in this paper was a particular case of VS isolated to the BA, that is to say, not affecting the vertebral arteries or the PCA. Another prominent study analyzing the comparative accuracies of angiography and TCD in the detection of vasospasm in both the anterior and posterior circulations highlighted an illustrative case of increased FVs in the BA of a patient ten days after aneurysmal SAH. ${ }^{28}$ Indeed other contemporaneous evaluations of the clinical applicability of TCD had included data on the posterior circulation; nevertheless, the scientific focus on the anterior circulation continued to predominate, so that VS of the VB system remained essentially uncharted territory. Although the criteria and technical parameters for insonation of the posterior circulation had been established years before. ${ }^{29,30}$ Sloan et al. ${ }^{31}$ in 1994 were the first to ascertain the sensitivity and specificity of TCD for capturing VS in both the basilar and vertebral arteries. In addition to confirming a direct correlation between severity of stenosis grade and mean FVs in the VB system, as previously had been proven with regard to the circle of Willis, they found that defining angiographic VS in mild to moderate cases by convention of FVs above $60 \mathrm{c} / \mathrm{m}$ (nearly twice the normal value) resulted in a sensitivity and specificity for the vertebral arteries of $44 \%$ and $87.5 \%$ respectively, and $76.9 \%$ and $79.3 \%$ for the BA, respectively. However, they observed that when the diagnostic criterion was elevated to $\geq 80 \mathrm{~cm} / \mathrm{s}$ for the vertebral artery, and $\geq 95 \mathrm{~cm} / \mathrm{s}$ for the BA, all false-positive results were eliminated, consequently providing a much improved specificity and a positive predictive value of $100 \%$. Since the sensitivity of the test thereby was compromised, and having attributed a high false positive rate to factors such as increased collateral flow and hyperemia, Sloan et al. ${ }^{31}$ stressed the necessity of devising a posterior circulation FV ratio analogous to the "Lindegaard ratio," as described above, and so opened the door to further research to lay the foundation for accurate TCD evaluation of VB vasospasm. Though Lee et al. ${ }^{32}$ attempted to improve TCD diagnosis of vasospasm by aid of an "initial slope index" (ISI) that previously had been proposed as an alternative to the Lindegaard's Index (by accounting for anatomical variations in the internal carotid arteries), ${ }^{33}$ its ability to compensate for elevated FVs in the BA was undermined by its exclusion of cerebral blood flow within the posterior circulation. However, Soustiel et al. ${ }^{34}$ effectively solved this problem by formulating a novel intracranial/extracranial (IC/EC) FVs ratio for the posterior circulation. Of note, the BA/EVA and IVA/EVA FV ratios effectively were unchanged in SAH patients without evidence of VS on computerized tomography (CT) angiography in comparison to healthy subjects, whereas the BA/extracranial (EC) vertebral artery (VA) FVs ratio showed a $58.8 \%$ increase in patients with a stenotic BA diameter $<3 \mathrm{~mm}$, constituting a statistically significant and indeed virtually linear correlation with vasospasm severity; similar findings with respect to the vertebral arteries were achieved. This correlation was even further improved by replacing the BA diameter with its expression as a percentage of the average EC-VA diameter, thereby minimizing the anatomical variations in the size of the VAs that are liable to distort results of TCD measurements of FVs in the VAs)

\section{TCD Criteria for Posterior Circulation CVS}

In comparative analysis between $\mathrm{CT}$ angiography and $\mathrm{TCD}$ results Soutiel et al. ${ }^{34}$ reported showed that the BA/EC-VA FVs ratio was 2 in all patients with BA vasospasm (sensitivity $100 \%$ ), with a ratio of 3 implicating severe angiographic BA vasospasm $(50 \%$ diameter reduction). And so, just as Lindegaard et al. ${ }^{28}$ had done for the anterior circulation, Soustiel et al. ${ }^{34}$ innovated the use of an intracranial(IC)/ EC FVs ratio that significantly improved the accuracy of TCD in the diagnosis of BA-VS, and by doing so reduced the number of falsepositive results. Concerns about the accuracy of this new IC/EC index in light of certain methodologic irregularities, were put to rest by later analysis that elaborated on the biostatstical parameters for TCD diagnosis of BA-VS. Sviri et al ${ }^{35}$ evaluated Forty-three patients with aneurysmal SAH who underwent cerebral angiography during the acute phase of vasospasm (days 4-12 after the hemorrhage). BA narrowing was measured and compared to a baseline angiogram done within 48 hours from the initial hemorrhage. TCD was done within 6 hours prior to angiography and both BA and EC-VA mean FVs were measured: The BA/EC vertebral arteries FVs ratio was found to be in significant correlation with the degree of basilar artery narrowing $(\mathrm{p}<0.0001, \mathrm{r}=0.7792)$. A ratio higher than 2.0 was found in 28 of 31 patients with BA-VS. This ratio was associated with $90 \%$ sensitivity, $50 \%$ specificity and $85 \%$ positive predictive value (PPV). A ratio higher than 2.5 was found in 20 of 21 patients with BA narrowing of more than $25 \%$. A ratio higher than 3.0 was associated with $100 \%$ sensitivity for BA narrowing of more than $40 \%$. those TCD criteria (Table 1) for BA-VS remain the most relevant and widely accepted guidelines for TCD diagnosis for spasm within the posterior cerebral circulation. $^{36}$

Table I TCD Criteria for severity of basilar artery vasospasm; Basilar artery mean flow velocities (BA- MFVs); extracarnial/intracranial (EC/IC)

\begin{tabular}{lll}
\hline BA Vasospasm Severity & BA- MFVs (cm/sec) & EC/IC Ratio \\
\hline None & $<70$ & $<2.0$ \\
Mild (<20\% narrowing) & $70-79$ & $>2.0$ \\
Moderate (20-39\% narrowing) & $>80$ & $2.0-3.0$ \\
Significant $(>40 \%$ narrowing) & $>80$ & $>3$ \\
\hline
\end{tabular}

\section{Vertebrobasilar Vasospasm, Cerebral Hypoperfusion and Outcome after Aneurysmal Subarachnoid Hemorrhage}

With regard to the clinical outcomes of vasospasm of the posterior circulation, Lee et al..$^{32}$ had demonstrated with TCD measurements of blood flow in a group of patients with VS in the VB system (nearly all of whom had concomitant VS in the middle cerebral artery), thus affected in both the anterior and posterior circulations VS ended up 
with drastically worse clinical outcomes than those patients without TCD-detected VS in MCA only $(\mathrm{p}=0.013)$. Furthermore, in a study that revealed an overall incidence of VB-VS of $23.8 \%$ detected on TCD (that corresponded with Sloan's finding of 30.9\%) Soustiel et al. ${ }^{37}$ made a host of significant novel clinical observations, including that DCI, particularly with regard to brainstem impairment, was nearly three times more frequent in patients with TCD evidence for VB-VS than in those without. VB VS was significantly more frequent in association with traumatic SAH and FVs in the VB system closely correlated with the severity of the SAH (especially in trauma, perhaps owing to the frequent involvement of the midbrain and the brain stem). Moreover, Soustiel et al. ${ }^{37}$ noted that, whereas FVs in the anterior cerebral vessels do not correlate with the distribution of subarachnoid blood, in the VB system FVs were significantly elevated in patients with posterior basal cistern hemorrhage. In another report, Soustiel et al. ${ }^{38}$ revealed that severe BA VS was associated with permanent neurological deficit or death in $85.3 \%$ of a patient cohort. Ultimately, in concert with the findings of Lee et al. ${ }^{32}$ the Soustiel group ${ }^{38}$ clarified that FVs in the BA have unique clinical significance, whereby VS of the posterior circulation was associated with poor functional outcomes, suggesting that BA-VS may result in secondary insult to the brainstem. Indeed, subsequent research on the hemodynamics of BA-VS designed to explore that very thesis provides the basis for current knowledge on the neuroanatomic determinants of clinical outcomes associated with spasm in the VB system. In similar fashion to previous efforts that shed light on the correlation between regional hypoperfusion and DCI in the anterior circulation. ${ }^{39}$ Sviri et al. ${ }^{40,41}$ ascertained a causal link between basilar artery vasospasm and hypoperfusion to the brainstem and other areas supplied by the posterior circulation. By assessing a cohort of aneurysmal SAH patients with TCDs, baseline and follow-up (99m) Tc ethyl cysteinate dimer single-photon emission computed tomography (99mTc ECD-SPECT) imaging of the territories of the posterior circulation, and correlating the results to clinical evaluations of neurological status per the Glasgow Coma Score and Fisher's grading scale, it was found that $79.3 \%$ of cases of delayed brainstem hypoperfusion had TCD measurements consistent with BA VS. Moreover, severe hypoperfusion was discerned in all of the patients presenting with BA mean FVs exceeding $115 \mathrm{~cm} / \mathrm{s}$, thus providing a diagnostic threshold to identify patients at high risk for brainstem ischemia. In essence, delayed brainstem ischemia was found to be associated with higher bleeding intensity and elevated BA FVs, delayed ischemic neurological deficits, and so worse overall outcomes, which they posited could be secondary to significant compromise in flow through the perforating arteries emerging from the basilar artery at a $90 \%$ angle that serve as the main source of blood flow to the brainstem. In an additional research of Sviri et al. ${ }^{42} \mathrm{BA}$ narrowing $\geq 25 \%$ was found in 23 of 65 patients, and delayed brain stem (BS) hypoperfusion, as estimated by ECD-SPECT, was found in 16 . Fourteen of 23 patients with BA narrowing $\geq 25 \%$ experienced BS hypoperfusion, whereas only 2 of 42 patients with $\geq 25 \%$ BA narrowing experienced BS ischemia. Stepwise logistic regression after adjusting for age with Hunt and Hess grade, Fisher grade, hydrocephalus, and aneurysmal location as co-variables revealed BA narrowing $\geq 25 \%$ and delayed BS hypoperfusion to be significantly and independently associated with unfavorable 3 -month outcome $(P=0.0001$; odds ratio, 10.1 ; $95 \% \mathrm{CI}, 2.5$ to 40.8 ; and $P=0.007$; odds ratio, $13.8,95 \% \mathrm{CI}$ 2.18 to 91.9 , respectively) and was an independent prognostic factor highly associated with an unfavorable outcome in aneurysmal SAH patients with clinically suspected severe VS, further suggesting that endovascular therapy intervention should be considered in the event of significant spasm in the VB system. ${ }^{41,42}$

\section{Conclusion}

The incidence of VS after aSAH is high and is associated with increased morbidity and mortally rate. VS usually affect the anterior circulation and peeking on days 5-14 after the hemorrhage. The diagnosis of VS is based on clinical presentation, TCD evaluation and perfusion imagines. The incidence of posterior circulation VS and BAVS are lower than the incidence in the anterior circulation, however it is associated with worst outcome. The diagnosis of posterior circulation and BA VS is mainly based on measurement of TCD IC/ EC FVs ratio that have been correlated with arterial narrowing on CT angiography, cerebellar hypoperfusion and outcome. Within the past decade, there has been very little scientific activity to follow up on the research outlined above, However, TCD grading criteria for BA VS are in widely used on daily clinical practice for the diagnosis of BA vasospasm.

\section{Acknowledgements}

None.

\section{Conflicts of interest}

None.

\section{References}

1. Connolly ES Jr, Rabinstein AA, Carhuapoma JR, et al. Guidelines for the management of aneurysmal subarachnoid hemorrhage: a guideline for healthcare professionals from the American Heart Association/ American Stroke Association. Stroke. 2012;43(6):1711-1737.

2. Cossu G, Messerer M, Oddo M, et al. To look beyond vasospasm in aneurysmal subarachnoid hemorrhage. Biomed Res Int. 2014;2014:628597.

3. Grunwald IQ, Kühn AL, Schmitt AJ, et al. Aneurysmal SAH: current management and complications associated with treatment and disease. $J$ Invasive Cardiol. 2004;26(1):30-37.

4. Sakowitz OW, Santos E, Nagel A, et al. Clusters of spreading depolarizations are associated with aneurysmal subarachnoid hemorrhage. Stroke. 2013;44(1):220-223.

5. Cahill J, Calvert JW, Zhang JH. Mechanisms of early brain injury after subarachnoid hemorrhage. $J$ Cereb Blood Flow Metab. 2006;26(11):1341-1353.

6. Macdonald RL. Delayed neurological deterioration after subarachnoid haemorrhage. Nat Rev Neurol. 2014;10(1):44-58.

7. Sabri M, Lass E, Macdonald RL. Early brain injury: a common mechanism in subarachnoid hemorrhage and global cerebral ischemia. Stroke Res Treat. 2013;2013:394036.

8. Schubert GA, Seiz M, Hegewald AA, et al. Hypoperfusion in the acute phase of subarachnoid hemorrhage. Acta Neurochir Suppl. 2011;110(Pt $1): 35-38$.

9. Brilstra EH, Rinkel GJ, Algra A, et al. Rebleeding, secondary ischemia, and timing of operation in patients with subarachnoid hemorrhage. Neurology. 2000;55(11):1656-1660.

10. Schweizer TA, Al-Khindi T, Macdonald RL. Mini-mental state examination versus montreal cognitive assessment: rapid assessment tools for cognitive and functional outcome after aneurysmal subarachnoid hemorrhage. J Neurol Sci. 2012;316(1-2):137-140.

11. Springer MV, Schmidt JM, Wartenberg KE, et al. Predictors of global cognitive impairment 1 year after subarachnoid hemorrhage. Neurosurgery. 2009;65(6):1043-1050. 
12. Veldeman M, Höllig A, Clusmann H, et al. Delayed cerebral ischaemia prevention and treatment after aneurysmal subarachnoid haemorrhage: a systematic review. Br J Anaesth. 2016;117(1):17-40.

13. Vergouwen MD, Vermeulen M, van Gijn J, et al. Definition of delayed cerebral ischemia after aneurysmal subarachnoid hemorrhage as an outcome event in clinical trials and observational studies: proposal of a multidisciplinary research group. Stroke. 2010;41(10):2391-2395.

14. Schmidt JM, Wartenberg KE, Fernandez A, et al. Frequency and clinical impact of asymptomatic cerebral infarction due to vasospasm after subarachnoid hemorrhage. J Neurosurg. 2008;109(6):1052-1059.

15. Kassell NF, Sasaki T, Colohan AR, et al. Cerebral vasospasm following aneurysmal subarachnoid hemorrhage. Stroke. 1985;16(4):562-572.

16. Broderick JP, Brott TG, Duldner JE, et al. Initial and recurrent bleeding are the major causes of death following subarachnoid hemorrhage. Stroke. 1994;25(7):1342-1347.

17. Dorsch NWC, King MT. A review of cerebral vasospasm in aneurysmal subarachnoid haemorrhage. Part I: Incidence and effects. J Clin Neurosci. 1994;1(1):19-26.

18. Petruk KC, West M, Mohr G, et al. Nimodipine treatment in poor-grade aneurysm patients. Results of a multicenter double-blind placebocontrolled trial. J Neurosurg. 1988;68(4):505-517.

19. Al-Mufti F, Roh D, Lahiri S, et al. Ultra-early angiographic vasospasm associated with delayedcerebral ischemia and infarction following aneurysmal subarachnoid hemorrhage. $J$ Neurosurg. 2017;126(5):1545-1551.

20. Phan K, Moore JM, Griessenauer CJ, et al. Ultra-Early Angiographic Vasospasm After Aneurysmal Subarachnoid Hemorrhage: A Systematic Review and Meta-Analysis. World Neurosurg. 2017;102:632-638.

21. Crompton MR. Pathogenesis of cerebral infarction following the rupture of cerebral berry aneurysms. Brain. 1964;87:491-510.

22. Wilkins RH, Alexander JA, Odom GL. Intracranial arterial spasm: A clinical analysis. J Neurosurg. 1968;29(2):121-134.

23. Saito I, Ueda Y, Sano K. Significance of vasospasm in the treatment of ruptured intracranial aneurysms. J Neurosurg. 1997;47(3):412-429.

24. Saito I, Shigeno T, Aritake K, et al. Vasospasm assessed by angiography and computerized tomography. J Neurosurg. 1979;51(3):466-475.

25. Marshall LF, Bruce DA, Bruno L, et al. Vertebrobasilar spasm: A significant cause of neurological deficit in head injury. $J$ Neurosurg. 1978;48(4):560-564.

26. Aaslid R, Markwalder TM, Nornes H. Noninvasive transcranial Doppler ultrasound recording of flow velocity in basal cerebral arteries. $J$ Neurosurg. 1982;57(6):769-774.

27. Aaslid R, Huber P, Nornes H. Evaluation of cerebrovascular spasm with transcranial Doppler ultrasound. J Neurosurg. 1984;60(1):37-41.

28. Lindegaard KF, Bakke SJ, Aaslid R, et al. Doppler diagnosis of intracranial artery occlusive disease. J Neurol Neurosurg Psychiatry. 1986;49(5):510-518.
29. Grolimund P, Seiler RW, Aaslid R, et al. Evaluation of Cerebrovascular Disease by Combined Extracranial and Transcranial Doppler Sonography. Stroke. 1987;18(6):1018-1024.

30. DeWitt LD, Wechsler LR. Transcranial Doppler. Stroke. 1988;19:915-921.

31. Sloan MA, Burch CM, Wozinak MA, et al. Transcranial Doppler Detection of Vertebrobasilar Vasospasm Following Subarachnoid Hemorrhage. Stroke. 1994;25(11):2187-2197.

32. Lee JH, Martin NA, Alsina G, et al. Hemodynamically significant cerebral vasospasm and outcome after head injury: a prospective study. J Neurosurg. 1997;87(2):221-233.

33. Jakobsen M, Enevoldsen E, Dalager T. Spasm index in subarachnoid haemorrhage: consequences of vasospasm upon cerebral blood flow and oxygen extraction. Acta Neurol Scand. 1990;82(5):311-320.

34. Soustiel FJ, Shik V, Shreiber R, et al. Basilar Vasospasm Diagnosis: Investigation of a Modified"Lindegaard Index" based on Imaging Studies and Blood Velocity Measurements of the Basilar Artery. Stroke. 2002;33(1):72-77.

35. Sviri GE, Ghodke B, Britz GW, et al. Transcranial Doppler Grading Criteria for Basilar Artery Vasospasm. Neurosurgery. 2006;59(2):360-366.

36. Kinkaid MS, Souter MJ, Treggiari MM, et al. Accuracy of transcranial Doppler ultrasonography and single-photon emission computed tomography in the diagnosis of angiographically demonstrated cerebral vasospasm. J Neurosurg. 2009;110(1):67-72.

37. Soustiel FJ, Bruk B, Shik B, et al. Transcranial Doppler in Vertebrobasilar Vasospasm after Subarachnoid Hemorrhage. Neurosurgery. 1998;43(2):82-291.

38. Soustiel JF, Shik V, Feinsod M. Basilar Vasospasm Following Spontaneous and Traumatic Subarachnoid Haemorrhage: Clinical Implications. Acta Neurochir (Wien). 2002;144(2):137-144.

39. Davis SM, Andrews JT, Lichtenstein M, et al. Correlations between cerebral arterial velocities, blood flow, and delayed ischemia after subarachnoid hemorrhage. Stroke. 1994;23(4):492-497.

40. Sviri GE, Lewis DH, Correa R, et al. Basilar artery vasospasm and delayed posterior circulation ischemia after aneurysmal subarachnoid hemorrhage. Stroke. 2004;35(8):1867-1872.

41. Sviri GE, Britz GW, Lewis DH, et al. Brainstem hypoperfusion in severe symptomatic vasospasm following aneurysmal subarachnoid hemorrhage: role of basilar artery vasospasm. Acta Neurochir (Wien). 2006;148(9):929-934.

42. GE Sviri, DW Newell, Lewis DH, et al. Impact of Basilar Artery Vasospasm on Outcome in Patients with Severe Cerebral Vasospasm After Aneurysmal Subarachnoid Hemorrhage. Stroke. 2006;37(11):2738-2743. 